\title{
POLYCNEMUM VERRUCOSUM (AMARANTHACEAE), FIRST RECORD FOR THE ITALIAN FLORA AND COMPARISON WITH RELATED SPECIES P. ARVENSE
}

\author{
Duilio IAMONICO ${ }^{1}$
}

\begin{abstract}
Polycnemum verrucosum A. F. Láng is recorded for the first time for Italy (Lazio region, Central Italy). Morphological and ecological characteristics of this species, as compared with the related $P$. arvense L., are presented and discussed. Taxonomical notes are also provided.

Key words: distribution area, Italy, new record, Polycnemum L.

Izvleček

Vrsto Polycnemum verrucosum A. F. Láng smo prvič zabeležili na ozemlju Italije (regija Lazio, srednja Italija). Prikazali smo morfološke in ekološke značilnosti vrste v primerjavi s sorodno vrsto P. arvense L. Podali smo tudi taksonomske opombe.

Ključne besede: razširjenost, Italija, nova najdba, Polycnemum L.
\end{abstract}

\section{INTRODUCTION}

Polycnemum L. is a genus of $6-8$ species distributed in Europe, central-western Asia, northern Africa and northern America (Ball 1993, Gelin et al. 2003, Greuter et al. 1984, Shultz 2004).

Polycnemum (and the related genera Nitrophila S. Watson and Hemichroa R. Brown) is included in Polycnemoideae Raf. Placement and rank of this taxon has long been controversial and it was included in the family of Amaranthaceae Juss. (e. g. Boissier 1879, Soriano 1944), Chenopodiaceae Vent. (e. g. Bentham \& Hooker 1880, Kühn et al. 1993) or Caryophyllaceae Juss. (e. g. Moquin-Tandon 1837) or considered as morphological intermediates between Amaranthaceae and Chenopodiaceae (Volkens 1887) and between Paronochyeae and Caryophyllaceae (Aellen 1960-1961). Recent phylogenetic studies (Kadereit et al. 2003) clearly shown the affinity of Polycnemoideae with the genera traditionally included in the Amaranthaceae (e. g. Achyranthes
L., Amaranthus L., Gomphrena L.). From the morphological point of view, Polycnemoideae and Amaranthaceae are characterized in having petaloid tepals (white or pigmented, scarious or papyraceous) and stame filaments united into a filament tube [in Chenopodiaceae s. s. tepals are sepaloid (green and herbaceous) and the filaments are not united into a filament tube]. Moreover, Polycnemum has one character of Gomphrenoideae Kostel. (absent in Chenopodiaceae): 2-locular anthers.

At present, 5 species are recorded in Europe: Polycnemum arvense L., P. fontanesii Durieu \& Moq., P. heuffelii A. F. Láng, P. majus A. Brown, P. verrucosum A. F. Láng (Ball 1993, Uotila 2011), of which three (P. arvense, $P$. majus and $P$. heuffelii) are indicated for Italy (Conti et al. 2005, Conti et al. 2007, Iamonico 2011a).

In this paper $P$. verrucosum is reported for the first time for Italy (Lazio region). Taxonomical, morphological and ecological notes are provided as well as the delimitation in comparison with the related $P$. arvense.

\footnotetext{
${ }^{1}$ Department DATA, Section Environment and Landscape, University of Rome Sapienza, Via Flaminia 72, 00196-Rome, Italy. d.iamonico@yahoo.it
} 
The work is part of a study carried out by the author on the family of Amaranhtaceae s. 1. in Italy (e. g. Iamonico 2010, 2011b, 2012a, 2012b, Iamonico \& Jarvis 2012a, 2012b) and on the genus Polycnemum in particular, for the New Italian Flora (editor S. Pignatti, in prep.).

\section{MATERIAL AND METHODS}

This paper is based both on the analysis of the relevant literature and the examination of the specimens ( $P$. arvense, $P$. verrucosum) kept in the Herbaria BP, FI, LINN and RO.

The identification of both species were made using the protologues (Láng 1828, Linnaeus 1753) and the descriptions in the Flora Europaea (Ball 1993).

A morphological analysis of the exsiccata, based on 15 characters (12 quantitative and 3 qualitative - Table 1) was performed. The data matrix was processed by means of the software NCSS 2007 (Hintze 2007). The variability of the characters is illustrated by box plots and scatter plots.

Table 1: Characters measured (those labelled with an asterisk are qualitative).

Tabela 1: Merjeni znaki (zvezdica označuje kvalitativne znake).

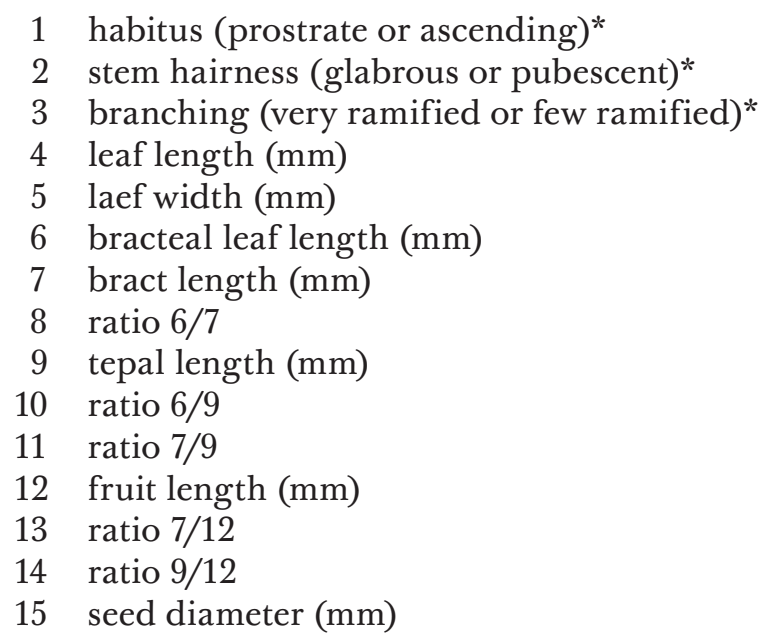

\section{RESULTS AND DISCUSSION}

The statistical analysis shows a clear separation between the species involved in the study, using the character No. 10 (see Table 1). The others ones have not statistical significance, showing partial (characters Nos. 6, 7, 8, 11,13) or total overlap of the measurements (characters Nos. 1, 2, 3, 4, 5, 9, 12, 14, 15) (Fig. 1).

Polycnemum verrucosum A. F. Láng, Syll. Pl. Nov. 1: 179 (1824).

= P. arvense L. var. verrucosum (A. F. Láng) Oborny, Fl. Mähr.: 334 (1885).

Annual (therophyte), to $50 \mathrm{~cm}$. Stem ascendent (sometimes erect), branched, glabrous or slightly pubescent. Leaves linear $(0.5-1.0 \times 3.0-12.0 \mathrm{~mm})$, plane-shaped, glabrous (sometimes slightly pubescent), pointed at the apex, usually glaucous. Inflorescence in terminal leafy spikes; bracteal leaves ovate-lanceolate (3-5 mm long) 2-3 times longer than the perianth, acute; bract of the flowers 2, with lateral membranous borders thinning to apex hyaline, white, 1.1-2 times longer than the perianth; tepals 5 , ovate, acute or acuminate (1.5$1.8 \mathrm{~mm}$ long); fruit ellipsoidal $(1 \times 1.3 \mathrm{~mm})$, subequal to the tepals, indehiscent; seeds lenticular (1-1.5 $\mathrm{mm}$ in diameter), black, fine sculptured.

Iconography: Aellen (1960-1961).

Chromosome number: unknown.

Taxonomical notes: Polycnemum verrucosum was first described from Hungary in 1824 “... in plagis arenososis, aridis, comitatus Pestiensis, Cumaniae, Faszygiae" (Láng 1824). MoquinTandon (1840) quoted the genus Halimocnemis C. A. Mey. in which Polycnemum was included, but the species $P$. verrucosum was not reported. De Candolle (1849) indicates the species in "... in arenosis Hungarice ..." and provided an extensive description. Oborny (1885) recognized the taxon at variety rank, under $P$. arvense. The comprehensive European floras (Greuter et al. 1984, Ball 1993) accepted $P$. verrucosum as separate species.

Ecology: Field, sandy and gravelly places (Ball 1993).

Flowering time: September to December.

General distribution: Austria, Czech Republic, Germany, Hungary, former Jugoslavia, Romania, Western and Eastern Russia, Turkey (Greuter et al. 1984, Jalas \& Souminen 1980, Ball 1993) and Italy. The Italian record extends to south-western the distribution area of P. verrucosum.

Distribution in Italy: no former Italian floras reported P. verrucosum [from Bertoloni (1854) to Conti et al. (2005)]. The specimens examined represent the first records for Italy (Lazio region, Central Italy). 

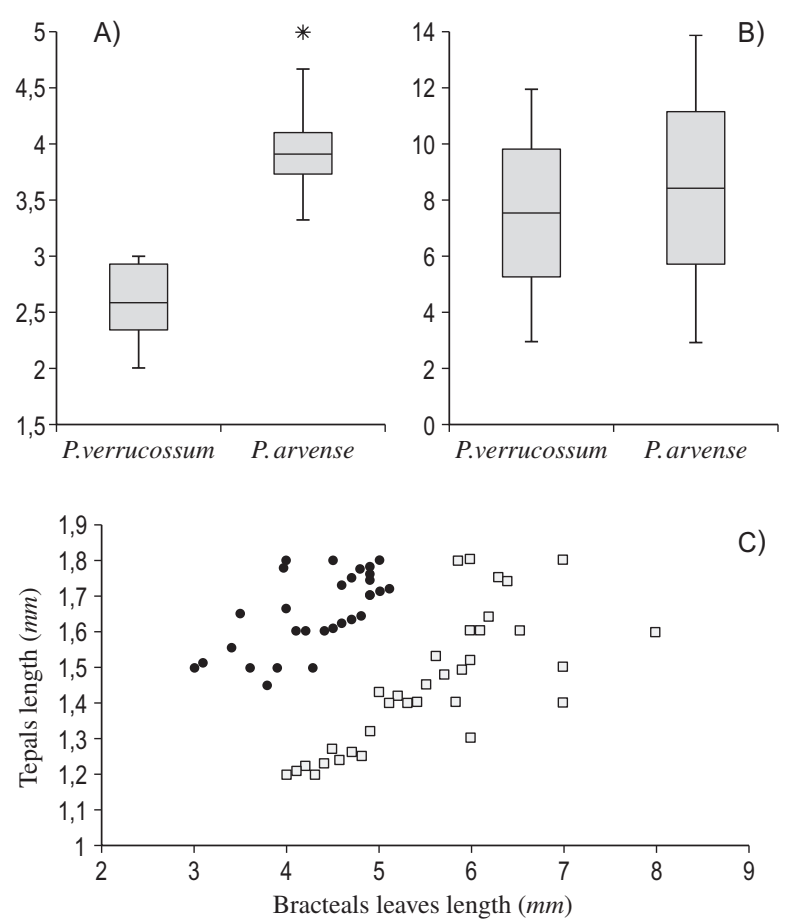

Figure 1: Box Plots (A: ratio bracteal leaves length/tepals length; B: leaves length) and scatter plot (D: bracteal leaves vs tepals; black circles for $P$. verrucosum, white squares for $P$. arvense) illustrating the variability of some characters.

Slika 1: Graf škatla z ročaji (A: razmerje braktealni list/dolžina tepala; B: dolžina lista) in razsevni diagram (D: braktealni listi proti tepalom; polni krožci za $P$. verrucosum, prazni kvadrati za $P$. arvense), ki prikazujeta variabilnost nekaterih znakov.

Polycnemum arvense L., Sp. Pl. 1: 35 (1753).

Annual (therophyte), to $50 \mathrm{~cm}$. Stem ascendent (sometimes erect), branched, glabrous or verrucose. Leaves linear $(0.5-1.3 \times 3.0-14.0 \mathrm{~mm})$, plane-shaped, glabrous, pointed at the apex, not glaucous. Inflorescence in terminal leafy spikes; bracteal leaves ovate-lanceolate (4.5-8 $\mathrm{mm}$ long) at least 3 times longer than the perianth, acute; bract of the flowers 2 , with lateral membranous borders thinning to apex, hyaline, white, 1.1-2 times longer than the periath; tepals 5 , ovate-lanceolate, acute [1.2-1.7(-2) mm long]; fruit ellipsoidal $(1 \times 1.5 \mathrm{~mm})$, subequal to the tepals, indehiscent; seeds lenticular (1-1.7 $\mathrm{mm}$ in diameter), black or brown, fine sculptured.

Iconography: Gutiérrez Bustillo (1990).

Chromosome number: $2 \mathrm{n}=18$ (Tomšovic 1990), 24 (Gutiérrez Bustillo 1990).

Taxonomical notes: $P$. arvense was first described from Europe in 1753 “... in Gallaie, Italiae, Ger- maniae arvis ..." (Linnaeus 1753). De Candolle (1849) accepted the species rank for this species, but he pointed out an high intraspecific variability, reporting five varieties: $\alpha-$ multicaule Wallr., $\beta$ - roseum De Not., $\gamma-$ majus (A. Brown) DC., $\delta$ - recurvum Gaudin, $\varepsilon-$ pumilum (Hoppe ex Mert. \& W. D. J. Koch) DC. The diagnostic features for these taxa are: habitus, shape and colour of the leaves, ratio bracts/tepals, ratio fruit/tepal. The variability of these characters seems to be continue, except for the ratio bract/tepals that clearly distinguish the var. majus [De Candolle (1849) reported "Bracteae calycem vix superantes"], now accepted at specific rank (P. majus A. Brown).

Ecology: Human made habitat, such as uncultivated land or cultivated field, on dry soil (personal observation), at 0-1000 m a.s.l.

Flowering time: July to October.

General distribution: Albania, Austria, Bulgaria, Czech Republic, France, Germany, Greece, Hungary, Italy, former Jugoslavia, Romania, Russia, Spain, Switzerland and European Turkey (Greuter et al. 1984, Jalas \& Souminen 1980, Ball 1993).

Distribution in Italy (Conti et al. 2005, Conti et al. 2007, Iamonico 2012c, Iamonico \& Bovio 2012): Northern (old recorded in Valle d'Aosta region), Central and Southern Italy (Puglia excluded and old recorded in Campania and Calabria).

\section{CONCLUSIONS}

Extensive analysis of literature and careful Herbarium investigations allowed to record $P$. verrucosum in Italy for the first time, extending the distribution area of the species in Europe far to south-west, but it may represent an alien population the status of which is unknown so far and requires further investigations. The comparison with the related species $P$. arvense shows a clear separation between the two taxa on the basis of the ratio bracteal leaves length/tepals length.

\section{ACKNOWLEDGEMENTS}

I am grateful to Directors and Curators of all quoted Herbaria for their support during my visits, loan of specimens/photographs or request informations. 


\section{REFERENCES}

Aellen, P. L. 1960-1961: Chenopodiaceae. In: Hegi, G. (ed.): Illustrierte Flora von Mitteleuropa 3(2). Paul Parey, Berlin and Hamburg, pp. 533-747.

Ball, P. W. 1993: Polycnemum L. In: Tutin, T. G., Burges, N. A., Chater, A. O., Edmondson, J. R., Heywood, V. H., Moore, D. M., Valentine, D. H., Walters, S. M. \& Webb, D. A. (eds.): Flora Europea (second edition), 1. Cambridge University Press, Cambridge, 110 pp.

Bentham, G. \& Hooker, J. D. 1880: Genera plantarum, 1(1). Lovell Reeve, London, 454 pp.

Bertoloni, A. 1854: Flora italica, sistens plantas in Italia et in insulsi circumstandibus sponte nascentes, 1. Ex Typographaeo Haeredum Richardi Masii, Bologna, 883 pp.

Boissier, E. 1879: Flora Orientalis, 4. H. Georg., Genevae et Basileae, 1276 pp.

Conti, F., Abbate, G., Alessandrini, A. \& Blasi, C. (eds.) 2005: An annotated checklist of the Italian vascular flora. Palombi \& Partner, Roma, 420 pp.

Conti, F., Alessandrini, A., Bacchetta, G., Banfi, E., Barberis, G., Bartolucci, F., Bernardo, L., Bonacquisti, S., Bouvet, D., Bovio, M., Brusa, G., Del Guacchio, E., Foggi, B., Frattini, S., Galasso, G., Gallo, L., Gangale, C., Gottschlich, G., Grünanger, P., Gubellini, L., Iiriti, G., Lucarini, D., Marchetti, D., Moraldo, B., Peruzzi, L., Poldini, L., Prosser, F., Raffaelli, M., Santangelo, A., Scassellati, E., Scortegagna, S., Selvi, F., Soldano, A., Tinti, D., Ubaldi, D., Uzunov. D. \& Vidali, M. 2007: Integrazioni alla checklist della flora vascolare italiana. Natura Vicentina (2006) 10: 5-74.

De Candolle, A. 1849: Prodromus systematis naturalis regni vegetabilis, 13(2). Victoris Masson, Parisiis, 472 pp.

Gelin, Z., Mosyakin, S. L. \& Clemants, S. E. 2003: Polycnemum L. In: Wu, Z. Y., Raven, P. H. \& Hong, D. Y. (eds.): Flora of China, 5. St. Louis and Missouri Botanical Garden Press, Beijng, pp. 375-376.

Greuter, W., Burdet, H. \& Long, G. (eds.) 1984: Med-checklist. Pteridophyta, Gymnospermae, Dicotyledones (Acanthaceae-Cneoraceae), 1. Conservatoire et Jardin botanique de la Ville de Genéve, Geneve, 330 pp.

Hintze, J. 2007: NCSS 2007. NCSS, LLC. Kaysville. Gutiérrez Bustillo, A. M. 1990: Polycnemum L. In: Castroviejo, S., Laínz, M., López Gonzáles,
G., Monserrat, P., Muñoz Garmendia, F., Paiva, J. \& Villar, L. (eds.): Flora Iberica, 2. Real Jardín Botánico, Madrid, pp. 478-479.

Iamonico, D. 2010: Confirmation of the occurrence of Chenopodium strictum ssp. strictum (Amaranthaceae s. 1.) in Italy. Phyton (Horn, Austria) 49(2): 235-240.

Iamonico, D. 2011a: Polycnemum heuffelii Láng. In: Gretuer, W. \& Raus, T. (eds.): Med-Cheklist notulae 30. Willenowia 41(2): 314.

Iamonico, D. 2011b: Dysphania anthelmintica (Amaranthaceae), new to the non-native flora of Italy, and taxonomic considerations on the related species. Hacquetia 10(1): 41-48.

Iamonico, D. 2012a: Amaranthus powellii S. Watson ssp. cacciatoi comb. \& stat. nov. (Amaranthaceae). Nordic Journal of Botany 30(1): 12-16.

Iamonico, D., 2012b: Studies on the genus Atriplex L. (Amaranthaceae) in Italy. II. Lectotypification of Atriplex elongata Guss. (Amaranthraceae). Candollea 67(1): 181-185.

Iamonico, D. 2012c: Notula 1933. Polycnemum arvense L. Notulae alla checklist della flora vascolare italiana 14. Informatore Botanico Italiano (in press).

Iamonico, D. \& Bovio, M. 2012: Notula 1930. Polycnemum arvense L. Notulae alla checklist della flora vascolare italiana 14. Informatore Botanico Italiano (in press).

Iamonico, D. \& Jarvis, C. E. 2012a: Lectotypification of two Linnaean names in Chenopodium $\mathrm{L}$. (Amaranthaceae). Taxon 61(3): 864-865.

Iamonico, D. \& Jarvis, C. E. 2012b: Lectotypification of Linnaean names in the genus Celosia $\mathrm{L}$. (Amaranthaceae). Taxon 61(5): 1101-1102.

Jalas, J. \& Suominen, J. 1980: Atlas florae Europeae. Distribution of Vascular Plants in Europe. Helsinki University Printing House, Helsinki, 119 pp.

Kadereit, G., Borsch, T., Weising, K. \& Freitag, H. 2003: Phylogeny of Amaranthaceae and Chenopodiaceae and the evolution of C4photosynthesis. International Journal of Plant Science 164: 959-986.

Kühn, U., Bittrich, V., Carolin, R., Freitag, H., Hedge, I. C., Uotila, P. \& Wilson, P. G. 1993: Chenopodiaceae. In: Kubitzki, K. (ed.): Families and genera of vascular plants, 2. Springer, Berlin, pp. 253-281.

Láng, A. F. 1824: Specierum novarum, et varietatum notabiliorum in Hungaria detectarum descriptio. In: Hornschuch, C. F. (ed.): Sylloge plantarum novarum itemque minus cognitar- 
um a praestantissimis botanicis adhuc viventibus collecta et a societate regia botanica ratisbonesi edita. Typis Viduae C. E. Brench, Ratisbonae, pp. 179-180.

Linnaeus, C. 1753: Species Plantarum, 1. Laurentii Salvii, Stockholm, 560 pp.

Moquin-Tandon, A. 1837: Note sur le genre Polycnemum et sur une nouvelle tribu de la famille des Paronychees. Ann. Sci. Nat. 7: 33-42.

Moquin-Tandon, A. 1840: Chenopodearum monographica enumeratio. P.-J. Loss, Parisiis, 182 pp.

Oborny, A. 1885: Flora von Mährer in Österreich. Der Verein, Brünn.

Shultz, L. M. 2004: Polycnemum L. In: Flora of North America Editorial Committee (eds.) : Flora of North America North of Mexico, 4. Oxford University Press, New York \& Oxford, pp. 261-262.

\section{APPENDIX}

Specimina visa selecta.

Polycnemum verrucosum A. F. Láng - Hungary: Vas. In glareosis argillaceis ad pagum Ostfy, 20.IX.1916, Trautmann (BP); Vas. In agris pag. Perint, 30.IX.1922, $220 \mathrm{~m}$ a.s.l., A. Boros (BP); Zala. In agris ad vias ferrena prope opp. Nagykansea, $150 \mathrm{~m}$ a.s.l., 03.IX.1923, A. Boros (BP); Pest. Pest, in pascuis arenosis ad rivum Rákos ad Isaszeg, versus Gödöllö, 180 m a.s.l., 19.X.1930, A. Boros (BP); Fejér. Fejér, in argillosis proprter vias ad "Fatelep" prope Csákberény, $220 \mathrm{~m}$ a.s.l., 06.IX.1936, A. Boros (BP); BorsodAbauj-Zemplén. Erdöbénye, Zemplén., in rupibus andesiticis, 17.X.1948, leg. et det. G. Andreánzsky, rev. L. Felfönly, 28.V.1999 (BP). - Italy: Lazio. sub Polycnemum sp., Roma, Colli di Bravetta, 03.XII.1892, G. $B$. Canneva, rev. G. Lusina (sub P. arvense L.), rev. D. Iamonico, 07.IX.2011 (RO-HR); Trevignano, Monte Rocca Romana, 23.IX.1897, A. Pappi, rev. G. Lusina (sub P. arvense L.), rev. D. Iamonico, 07.IX.2011 (RO-HR); sub Polycnemum arvense L., Albano presso li Cappuccini, IX.1858, E. Rolli, rev. D. Iamonico, 07.IX.2011 (RO-HR); sub Polycnemum arvense L., all'Ospedaletto vicino Roma, 11.X.1859, E. Rolli, rev. D. Iamonico, 07.IX.2011 (RO-HR); ibidem; sub Polycnemum arvense L., presso la Macchia di Marco Simone, 26.X.1886, Mori, rev. D. Iamonico, 07.IX.2011 (ROHR); Prov. di Roma, Monte Migliore fuori Porta San Paolo, 21.XI.1899, R. Pirotta et E. Chiovenda, rev. G. Lusina (sub Polycnemum arvense L.), rev. D. Iamonico, 07.IX.2011 (RO-HR).

Polycnemum arvense L. - C. Linnaeus, sine die (LINN 55.2). C. Linnaeus, Hortus Upsaliensis, sine die (LINN 55.1). - Hungary: Sorki, in agris areno-
Soriano, A. 1944: El género Nitrophila en la Argentina y su posición sistemática. Revista Argentina de Agronomía 11: 302.

Tomšovic, P. 1990: Polycnemum L. - Chruplavník. In: Hejný, S. \& Slavík, B. (eds.): Kvētena České Republiky, 2. Slavomil Hejný \& Bohumil Slavík, Praha, pp. 215-219.

Uotila, P. 2011: Chenopodiaceae (pro major parte). In: Euro-Med Plantbase - the information resource for Euro-Mediterranean plant diversity. http://w2.bgbm.org/EuroPlusMed/ (last accessed 10 April 2012).

Volkens, G. 1887: Die Flora der Ägyptisch-arabischen Wüste. Borntrager, Berlin, 156 pp.

Received 23. 4. 2012

Revision received 8. 10. 2012 Accepted 10. 10. 2012

sis, 30.VII.1892, leg. et det. Martón, rev. L. Somlayay, 20.IX.2004 (BP); Györ-Moson-Sopror. Györ, in arenosis ad Ferenczháza puszta, 16.VII.1918, $100 \mathrm{~m}$ a.s.l., A. Boros (BP); Nògrád. Mt. Börzsöny, ad vias, $400 \mathrm{~m}$ a.s.l., 15.VII.1934, Z. Kárpáti (BP); Komárom-Esztergom. in rupestribus andesit. "Vadállókovek",monti Keserús prope pag. DÖMÖS, 400-500 m a.s.l., 08.VIII.1948, A. Boros (BP); Borsod-Abauj-Zemplén. prope pagum Füzér, in rupibus montis Füzérì-vár, Montes Zemplenenses, 21.IX.1955, A. Horánszky (BP); Pest. Dunakeszi, Homokos, ruderalis helyen, 30.IX.1957, leg. et det. Fárvorka-Csapodi, rev. L. Somlayay, 16.IX.2004 (BP); Pest. Prope pag. Parasa, in montibus Börzsöny, 31.VIII.1960, leg. et det. f. Lacza, rev. L. Felföldy, 16.II.1996 (BP); Pest. SzentendreIzbég, Visegrádi Mtz., Izbég felett, a Kis-Kö-Hegy keleti lejtöjén, kimosott andezittörmelékes földúton, 14.VIII.1999, E.I. Böhm, rev. L. Somlayay, 17.IX.2004 (BP); Pest. Visegrád, Ördög-Bánya, graminosis saxosis, 01.IX.1004, Z. Barina (BP). - Italy: Basilicata. Castelgrande (Potenza), pr. Cappella di S. Viro, in pendici arenaceo-argillose molto pascolate, 23.X.1935, G. Lusina (RO-HG). Emilia-Romagna. prov. di Bologna, M. Paderno, VII.1883, G. Pizzini (RO-HG). Lazio. Roma, Centocelle, Borraccia (tra questa e la stazione), 21.X.1956, B. Anzalone (RO-HA); sub Polycnemum arvense $\mathrm{L}$. var. majus (R. Br.) Fiori, Roma, prati a Cinecittà, 07.IX.1962, A. Cacciato, rev. D. Iamonico, 06.XII.2010 (RO-HR). Liguria. Genova, letto del torr. Sturla a levante, 24.VIII.1887, sine coll. (ROHG). Lombardia. Pavia, luoghi sterili sabbiosi intorno alla città, VII.1872, G. Gibelli (RO-HG). Piemonte. Torino, Parco del Valantiino, 04.IX.1899, Ferrari (RO-HG). Veneto. Verona, circum Verona, VIII.1876, A. Goiran (RO-HG). 